\title{
Compensation of detector solenoid effects on the beam size in a linear collider
}

\author{
Yuri Nosochkov and Andrei Seryi \\ Stanford Linear Accelerator Center, P.O. Box 20450, Stanford, California 94309, USA
}

(Received 29 July 2004; published 28 February 2005)

\begin{abstract}
In this paper, we discuss the optics effects of the realistic detector solenoid field on beam size at the interaction point (IP) of a future linear collider and their compensation. It is shown that most of the adverse effects on the IP beam size arise only from the part of the solenoid field which overlaps and extends beyond the final focusing quadrupoles. It is demonstrated that the most efficient and local compensation can be achieved using the novel method of weak antisolenoids near the IP, while a correction scheme which employs only skew quadrupoles is less efficient, and compensation with strong antisolenoids is not appropriate. One of the advantages of the proposed antisolenoid scheme is that this compensation works well over a large range of the beam energy.
\end{abstract}

\section{INTRODUCTION}

A linear collider (LC) must collide nanometer scale flat beams at the interaction point (IP) in order to reach the desired luminosity. The LC beam sizes are typically several nanometers in vertical and several hundred nanometers in horizontal plane. To produce these very small beam sizes requires strong optics and careful attention to any effects that can degrade the ideal beam, and, in particular, compensating the coupling and other distortions introduced by the detector solenoid.

In circular colliders, the standard methods of compensating for the detector solenoid include antisolenoids or skew quadrupoles or their combination. The simplest correction employs a pair of antisolenoids installed on either side of the detector solenoid. In this scheme of strong antisolenoids, the combined strength of the compensating and detector solenoids on each side of IP must vanish in order to cancel the beam tilt both at the IP and outside of the solenoid. This is automatically achieved if there are no other magnets inside the solenoid. The advantage of this scheme is that it is completely local and corrects all energies at once, but the drawback is that the antisolenoids do not permit the interaction region (IR) quadrupoles to be placed close to the IP. This limits the strength of the final beam focusing and hence the luminosity. This type of compensation was used in the early SPEAR and PEP [1] colliders and is presently used at KEK-B [2].

The strong antisolenoid method can be expanded to include quadrupoles inside the solenoid field. In this case, each quadrupole inside the field must be rotated by the same angle as the coupled beam would have at that quadrupole location. The tilted quadrupoles do not generate additional coupling, and hence the solenoidantisolenoid compensation is preserved. For ideal correction, however, the quadrupole frame would have to rotate continuously along the quadrupole length to follow the beam angle. For practical purposes, the continuous rotation can be replaced by an average tilt angle in each quadrupole and the remaining residual coupling minimized by small adjustment of the tilt angles as is done at DAFNE [3]. One disadvantage of this scheme is that operating at a different energy or different solenoid field requires a mechanical adjustment of the quadrupole tilt, which may be technically difficult.

Solenoid compensation using only skew quadrupoles has been implemented, for example, at the $e^{+} e^{-}$colliders LEP [4] and PEP-II [5]. In this scheme, local compensation of the coupling terms at the IP requires up to four skew quadrupoles on each side of the IP. The number of correctors may be reduced if the optics is symmetric about the IP or if some coupling terms can be neglected. Advantages of this method are that the skew quadrupoles are shorter than the antisolenoids, do not require space near the IP, can be adjusted for changing optics parameters, and can correct coupling coming from other sources. The drawback is that this correction, typically, is not as local as the strong antisolenoid scheme because the skew quadrupoles have to be placed at locations with specific phase advance from the IP. Consequently, the longer correction region in the real machine includes more magnet errors and, hence, requires a more careful implementation.

Compared to circular machines, the solenoid compensation in linear colliders has a few distinctive features: (1) correction is essential up to the collision point but may not be so stringent afterwards, (2) the IP beam focusing is much stronger than in existing rings, and (3) the optics must accommodate a large range of beam energy. For example, in the next generation linear collider, the IP must be designed to cover $90 \mathrm{GeV}$ to $1 \mathrm{TeV}$ in the center of mass. The standard methods of solenoid compensation used in circular accelerators are not appropriate for a linear collider. A novel method of weak antisolenoids appears to be the optimal compensation strategy.

This paper evaluates the effects of the detector solenoid field on the beam size and orbit at the IP of the Next Linear Collider (NLC). The study assumes the design optics of the NLC beam delivery system (BDS) with realistic mod- 
els of the detector solenoid field, provides both the analytical estimate and particle tracking calculation of the effects on the beam, and discusses possible methods of compensation.

All presented calculations are for the optics of the first interaction region in the NLC design [6] and for a beam energy of $250 \mathrm{GeV}$. The ideal rms beam sizes at the IP are $\sigma_{x 0}=243 \mathrm{~nm}$ and $\sigma_{y 0}=3 \mathrm{~nm}$, and beam divergencies $\sigma_{x p 0}=30.4 \mu \mathrm{rad}$ and $\sigma_{y p 0}=27.3 \mu \mathrm{rad}$. The energy spread has a two-horn distribution with the half-width of $0.4 \%$ and the effective rms value of $\sigma_{E} \approx 0.25 \%$. The IP beta functions are $\beta_{x 0}=8 \mathrm{~mm}, \beta_{y 0}=0.11 \mathrm{~mm}$, and there is a nonzero horizontal angular dispersion $\eta_{x 0}^{\prime}=$ 0.0094 at the IP. The latter is needed to create dispersion at the final doublet (FD) sextupoles for a local correction of the BDS chromaticity [7]. The optics of the complete BDS is shown in Fig. 1 and the optics near the IP is shown in Fig. 2. The free space between the IP and the final doublet
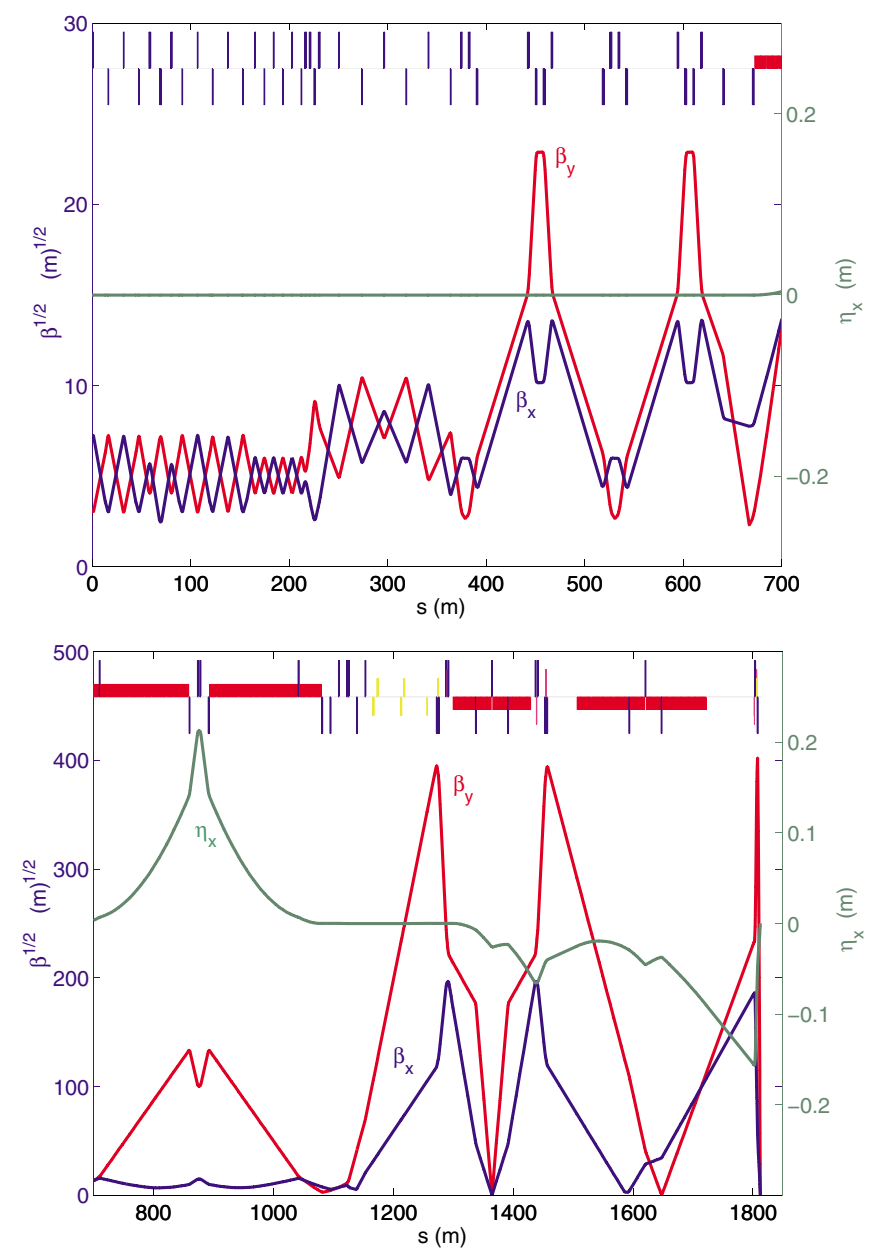

FIG. 1. (Color) Optics of the Next Linear Collider (NLC) beam delivery system. The IP is located at $s=1812.56 \mathrm{~m}$. A skew correction section is located in the first hundred meters. Correction sextupoles, whose displacements form the linear knobs, are at maxima of the beta function near $s=1270$ and $1450 \mathrm{~m}$ and in the FD.
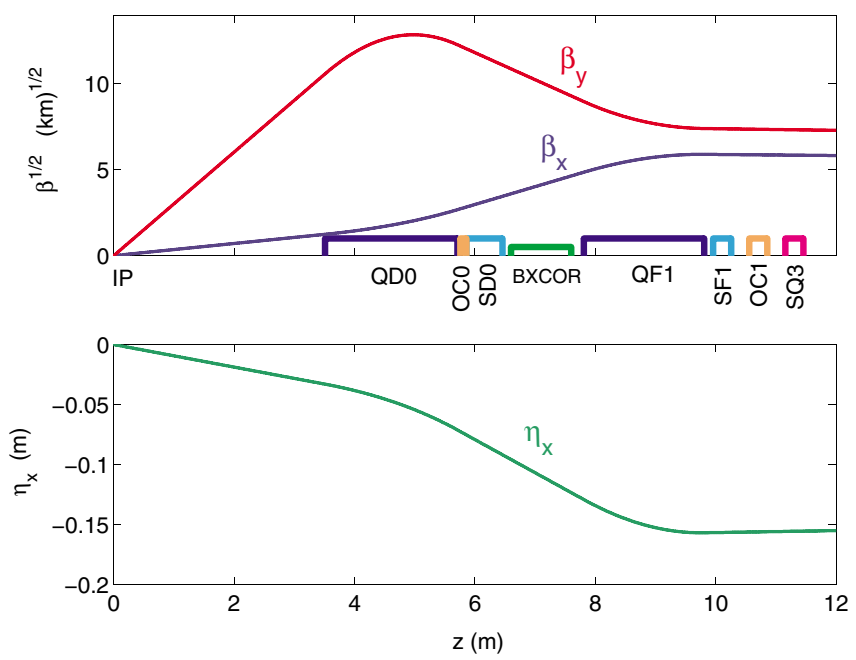

FIG. 2. (Color) Optics of the Next Linear Collider beam delivery system near the IP showing betatron functions (top) and horizontal dispersion (bottom). Locations of the final doublet magnets are shown, including quadrupoles QD0 and QF1, sextupoles SD0 and SF1, octupoles OC0 and OC1, skew quadrupole SQ3, and optional vertical corrector BXCOR. IP is at $z=0 \mathrm{~m}$.

is $L_{*}=3.51 \mathrm{~m}$, and the half crossing angle is $\theta_{c}=$ $10 \mathrm{mrad}$. These figures also show the locations of the final doublet quadrupoles, correcting sextupoles, octupoles and skew quadrupole near the IP, and the location of the skew correction section and sextupoles forming the linear knobs in the BDS. Note that the beam line in Fig. 2 is shown in the reversed direction, with the IP on the left side, for easier comparison with the ANSYS plot of detector solenoid field map. In all figures except Fig. 1 the IP location is at $z=0 \mathrm{~m}$.

Two realistic detector solenoid models were considered: the silicon detector ( $\mathrm{SiD}$ ) and the large detector (LD). The maximum field values are 5 and $3 \mathrm{~T}$, respectively, and the corresponding field integrals over half the detector are 16.7 and $14.4 \mathrm{~T} \mathrm{~m}$. Figure 3 shows the ANSYS model for the SiD detector and Fig. 4 shows the calculated longitudinal and radial components of the solenoid field in these models. Note that the LD field extends farther inside the FD quadrupoles.

For comparison with the above realistic models, a fictitious "tiny" solenoid model was also studied. This model was scaled from SiD and compressed longitudinally with the maximum field of $12 \mathrm{~T}$ and the field integral over half the detector of $18.0 \mathrm{Tm}$ as shown in Fig. 4. The essential feature of this model is that the solenoid is chosen to be short enough so that it does not overlap with the final doublet, in contrast to SiD or LD models.

The organization of this paper is as follows: Section II recapitulates the optics transformation in the basic hard edge solenoid model. Section III introduces the effects generated in the realistic solenoid models with overlapping solenoid field and final doublet quadrupoles. Section IV provides an analytic description of the coupling and orbit 


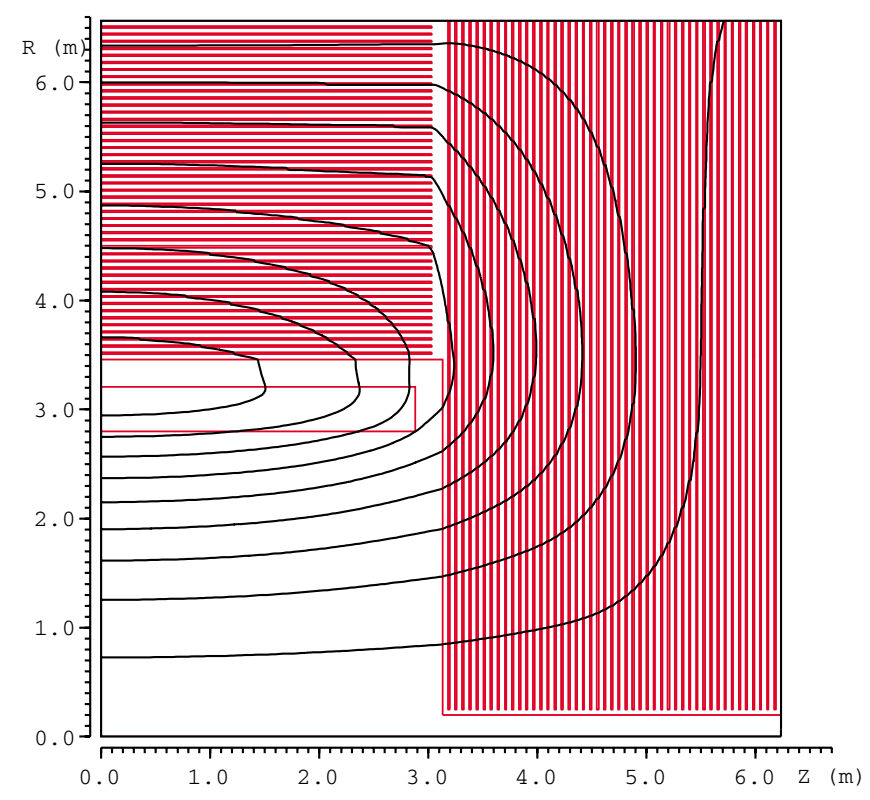

FIG. 3. (Color) The model of the silicon detector (SiD) with field lines calculated by ANSYS.

effects at the IP caused by the overlapped quadrupoles and solenoid, leading to the concept of weak antisolenoids for detector solenoid compensation. The application of the novel method of weak antisolenoids to SiD and LD compensation is discussed in Sec. V, where the optimized configurations of the antisolenoids and the results of compensation are presented. The enhancement of the solenoid effects at lower beam energies and performance of the weak antisolenoid method as a function of energy are discussed in the last part of Sec. V and compared with the skew quadrupole compensation method.
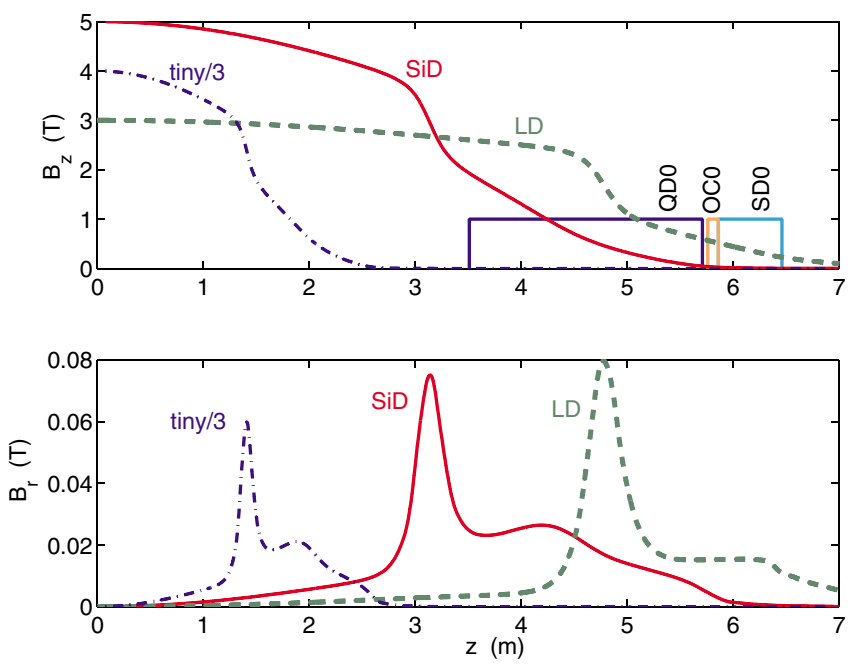

FIG. 4. (Color) Longitudinal and radial fields on the ideal beam trajectory with half crossing angle $\theta_{c}=10 \mathrm{mrad}$ for the $\mathrm{SiD}$, $\mathrm{LD}$, and tiny detectors. Locations of the final doublet elements are also shown. IP is at $z=0 \mathrm{~m}$.

\section{HARD EDGE SOLENOID MODEL}

Let us first describe the well-known properties of a simple hard edge solenoid with constant longitudinal field $B$ and length $\ell$. The first order matrix in this model can be written as $\hat{M}_{S}=\hat{M}_{E} \hat{M}_{L} \hat{M}_{E}{ }^{-1}$, where matrices $\hat{M}_{E}$ and $\hat{M}_{E}{ }^{-1}$ represent the integral effect of a linear radial field at the two solenoid edges and $\hat{M}_{L}$ is the transformation produced by the longitudinal field in the solenoid body (see, for example, [8]):

$$
\begin{gathered}
\hat{M}_{E}=\left(\begin{array}{cccc}
1 & 0 & 0 & 0 \\
0 & 1 & -K_{S} & 0 \\
0 & 0 & 1 & 0 \\
K_{S} & 0 & 0 & 1
\end{array}\right), \\
\hat{M}_{L}=\left(\begin{array}{cccc}
1 & S C / K_{S} & 0 & S^{2} / K_{S} \\
0 & C^{2}-S^{2} & 0 & 2 S C \\
0 & -S^{2} / K_{S} & 1 & S C / K_{S} \\
0 & -2 S C & 0 & C^{2}-S^{2}
\end{array}\right), \\
\hat{M}_{S}=\left(\begin{array}{cccc}
C^{2} & S C / K_{S} & S C & S^{2} / K_{S} \\
-S C K_{S} & C^{2} & -S^{2} K_{S} & S C \\
-S C & -S^{2} / K_{S} & C^{2} & C S / K_{S} \\
S^{2} K_{S} & -S C & -S C K_{S} & C^{2}
\end{array}\right) .
\end{gathered}
$$

Here, $K_{S}=B /(2 B \rho), S=\sin K_{S} \ell, C=\cos K_{S} \ell$, and $B \rho$ is the magnetic rigidity (equal to about $834 \mathrm{Tm}$ at $250 \mathrm{GeV}$ ). Note that the full solenoid matrix $\hat{M}_{S}$ is symplectic, but the individual matrices $\hat{M}_{E}$ and $\hat{M}_{L}$ do not satisfy this condition. This is because the matrices are written in terms of TRANSPORT variables $\left(x, x^{\prime}, y, y^{\prime}\right)$, while the symplecticity condition is derived and satisfied in the canonical system $\left(x, p_{x}, y, p_{y}\right)$. The two sets of variables are equal in a purely transverse field, hence the matrices of such magnets are automatically symplectic in a $\left(x, x^{\prime}, y, y^{\prime}\right)$ system. Inside a solenoid, however, there is a difference between the mechanical $\left(x^{\prime}, y^{\prime}\right)$ and canonical $\left(p_{x}, p_{y}\right)$ momenta created by the nonzero transverse components of the magnetic vector potential of a longitudinal field. Since the canonical transformation remains symplectic by definition, the transformation of $\left(x, x^{\prime}, y, y^{\prime}\right)$ inside a solenoid is no longer symplectic. This deviation arises only while inside a solenoid and should not be a concern [9].

Analysis of Eq. (3) shows that $\hat{M}_{S}$ can be expressed in a simpler form using a symplectic $2 \times 2$ matrix $\hat{F}$ :

$$
\hat{M}_{S}=\left(\begin{array}{cc}
C \hat{F} & S \hat{F} \\
-S \hat{F} & C \hat{F}
\end{array}\right), \quad \text { where } \hat{F}=\left(\begin{array}{cc}
C & S / K_{S} \\
-S K_{S} & C
\end{array}\right) \text {. }
$$

From here, it follows that $\hat{M}_{S}$ can be written as a product of an uncoupled matrix $\hat{M}_{F}$ and a rotation matrix $\hat{M}_{R}$, both being symplectic:

$$
\hat{M}_{S}=\hat{M}_{F} \hat{M}_{R}=\hat{M}_{R} \hat{M}_{F},
$$




$$
\hat{M}_{F}=\left(\begin{array}{cc}
\hat{F} & \hat{O} \\
\hat{O} & \hat{F}
\end{array}\right), \quad \hat{M}_{R}=\left(\begin{array}{cc}
C \hat{I} & S \hat{I} \\
-S \hat{I} & C \hat{I}
\end{array}\right),
$$

where

$$
\hat{I}=\left(\begin{array}{ll}
1 & 0 \\
0 & 1
\end{array}\right), \quad \hat{O}=\left(\begin{array}{ll}
0 & 0 \\
0 & 0
\end{array}\right) .
$$

Equations (5) and (6) clearly show that the solenoid coupling is just a rotation of the beam envelope in the $x-y$ plane by the angle $K_{S} \ell=B \ell /(2 B \rho)$. The uncoupled matrix $\hat{M}_{F}$ provides equal transformation in the $x$ and $y$ planes. In the approximation that the solenoid field is weak with $K_{S} \ell \ll 1$ (typically true for detector solenoids in accelerators), the $\hat{M}_{F}$ can be represented by a thin focusing lens surrounded by drifts of length $\ell / 2$ :

$$
\begin{aligned}
\hat{M}_{F}= & \left(\begin{array}{llll}
1 & \frac{\ell}{2} & 0 & 0 \\
0 & 1 & 0 & 0 \\
0 & 0 & 1 & \frac{\ell}{2} \\
0 & 0 & 0 & 1
\end{array}\right)\left(\begin{array}{cccc}
1 & 0 & 0 & 0 \\
-K_{S}^{2} \ell & 1 & 0 & 0 \\
0 & 0 & 1 & 0 \\
0 & 0 & -K_{S}^{2} \ell & 1
\end{array}\right) \\
& \times\left(\begin{array}{llll}
1 & \frac{\ell}{2} & 0 & 0 \\
0 & 1 & 0 & 0 \\
0 & 0 & 1 & \frac{\ell}{2} \\
0 & 0 & 0 & 1
\end{array}\right),
\end{aligned}
$$

where the focusing terms $-K_{S}^{2} \ell$ are equal in the $x$ and $y$ planes. Finally, taking into account that the rotation and drift matrices commute, a weak solenoid of length $\ell$ can be approximated by a thin solenoid with the matrix containing only the focusing and rotation terms as follows [8]:

$$
\hat{M}_{S}{ }^{\text {thin }}=\left(\begin{array}{cccc}
1 & 0 & K_{S} \ell & 0 \\
-K_{S}^{2} \ell & 1 & 0 & K_{S} \ell \\
-K_{S} \ell & 0 & 1 & 0 \\
0 & -K_{S} \ell & -K_{S}^{2} \ell & 1
\end{array}\right),
$$

and drifts of length $\ell / 2$ on either side. Note that in this approximation, $\hat{M}_{S}^{\text {thin }}$ is symplectic only to the first order of $K_{S} \ell$.

\section{SOLENOID EFFECTS WITH REALISTIC MODELS}

The situation becomes much more complicated for realistic models of the detector solenoid. The solenoid field is not constant along the longitudinal axis, the fringe field extends well inside the FD quadrupoles, and the solenoid is horizontally tilted with respect to the beam trajectory. Because preservation of the small vertical beam size at the IP is so essential for any linear collider, the most important effects to be identified and compensated are those that affect the vertical beam size.

The following discussions will often use the projection method [10], where beam aberrations originating at various locations in the lattice, for example, in the solenoid, are projected to the IP by an appropriate optics transformation.
The projected aberrations can be expressed in the form of matrix terms at the IP which add linearly, thus simplifying the analysis of their effects on the IP beam size. It is convenient to normalize the projected matrix terms (also referred here as beam correlations) to the nominal rms beam size at IP. This makes all the terms dimensionless for direct comparison of their effect on the beam size growth. For example, after normalization the projected matrix terms $R_{31}, R_{32}$, and $R_{36}$ become $\langle y x\rangle=R_{31} \frac{\sigma_{x 0}}{\sigma_{y 0}}$, $\left\langle y x^{\prime}\right\rangle=R_{32} \frac{\sigma_{x p 0}}{\sigma_{y 0}}$, and $\langle y E\rangle=R_{36} \frac{\sigma_{E}}{\sigma_{y 0}}$.

According to Eqs. (5) and (6), the vertical beam size growth in a solenoid is caused by the $\langle y x\rangle$ coupling term, and the rotation angle at IP is equal to $\approx B \ell /(2 B \rho)$, which is on the order of 0.01 in the NLC for $250 \mathrm{GeV}$ beam. Consequently, the vertical beam size would increase as $\frac{\sigma_{y}}{\sigma_{y 0}} \approx \sqrt{1+\left(\frac{\sigma_{x 0}}{\sigma_{y 0}} \frac{B \ell}{2 B \rho}\right)^{2}}$ yielding less than a factor of 2 of beam size growth at the IP.

It was also shown in Refs. [11-13] and can be verified using Eq. (9) that the geometric and chromatic orbit displacements generated when the beam passes through the solenoid at an angle are canceled at the IP as long as the collision point is located on the solenoid axis and the typically small transverse focusing due to the solenoid is neglected. For example, the vertical orbit deviates from the zero plane at the entrance to and along the solenoid, but returns exactly to zero at the IP in this approximation. This cancellation results from the fact that the solenoid radial and longitudinal fields have opposite effects on the beam orbit.

The above estimate, however, is correct only if there are no other magnets inside the solenoid (such as in the tiny model). Effects generated by the solenoid field extending into the final focus quadrupoles have been evaluated in several studies using numeric calculations with optics and tracking codes (see, for example, [5,13-16]). It was noted that the overlap of the solenoid and quadrupole fields gives rise to new coupling terms at the IP and significantly increases the vertical beam size. In particular, the $\left\langle y x^{\prime}\right\rangle$ correlation term at the IP [14] was observed to cause large beam size growth at SLC.

Tracking results for the realistic NLC detector solenoid models are presented in Fig. 5, which shows the beam phase space at the IP obtained using the DIMAD code [17] for three cases (SiD, tiny detector, and $\mathrm{SiD}$ with zero crossing angle). One can see that the vertical beam size growth is indeed about a factor of 2 for the tiny detector, but it is almost 2 orders of magnitude larger in the other three cases where the solenoid field extends into the FD quadrupoles. Figure 5 shows that the main contribution to the IP beam size is produced not by the $\langle y x\rangle$ term but by $\left\langle y x^{\prime}\right\rangle$ coupling when the solenoid and quadrupoles overlap. Additionally, as shown in Fig. 6, there is a nonzero vertical displacement of the beam at the IP. A quadrupole inserted inside the solenoid breaks the natural compensation of the 


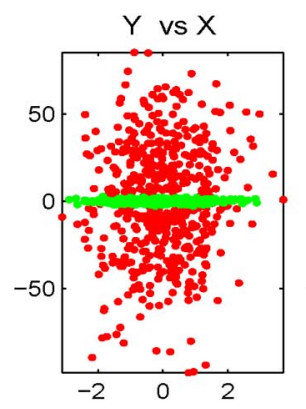

Silicon Detector, $20 \mathrm{mrad}$ crossing angle
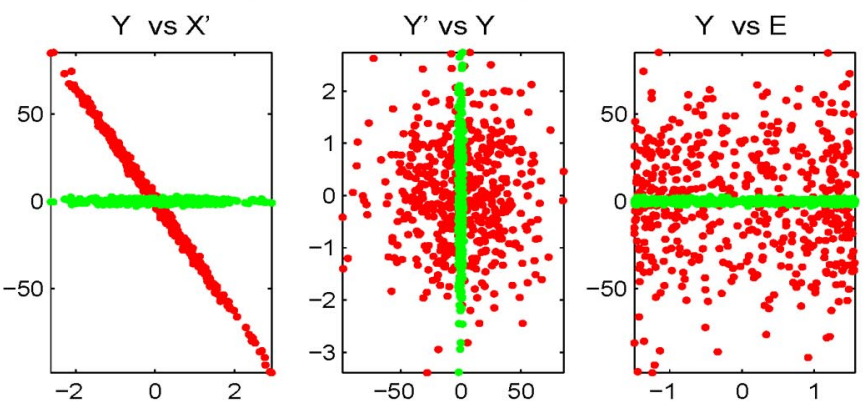

Silicon Detector, zero crossing angle
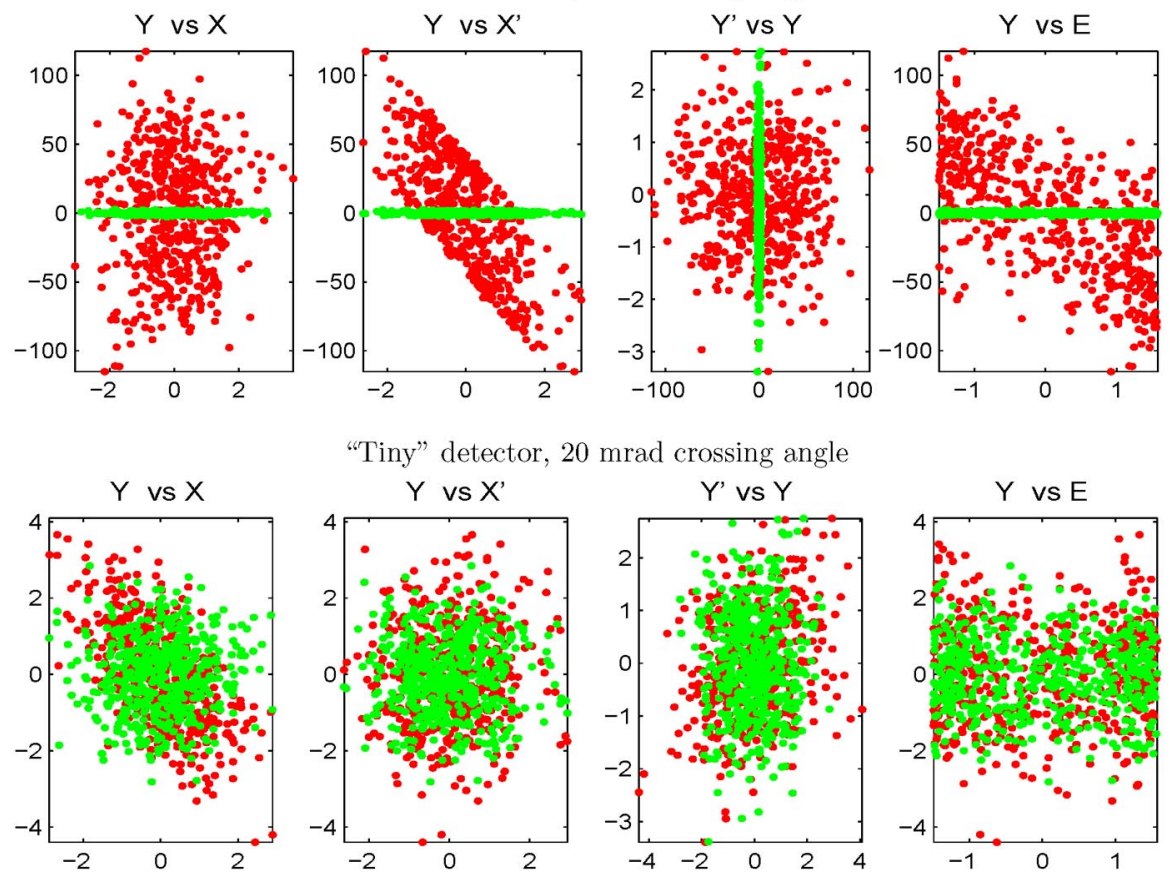

FIG. 5. (Color) Beam phase space at the IP obtained by tracking with two different detector solenoid models. Green dots show the ideal beam (no detector field); red, with detector solenoid field. Coordinates are normalized to the ideal beam sizes. No correction of the beam size was applied.
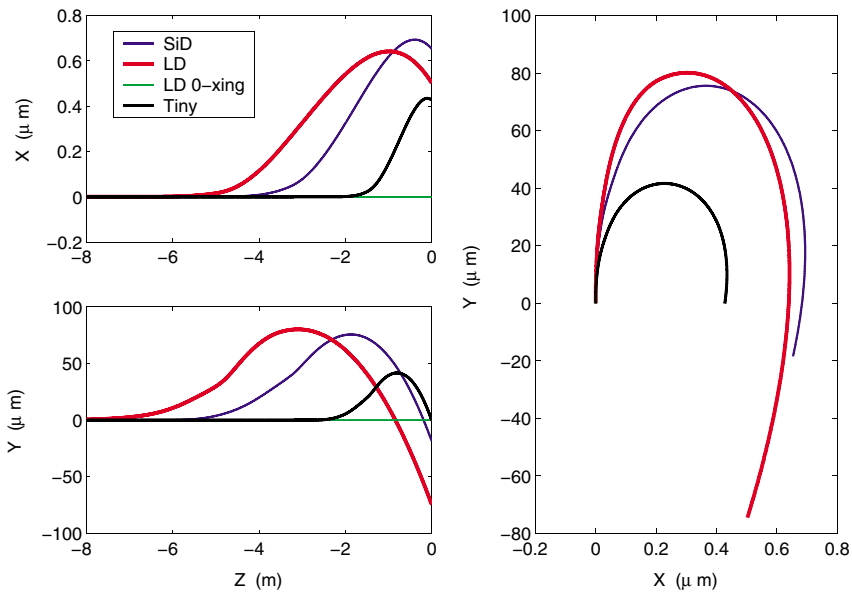

FIG. 6. (Color) Beam orbits obtained by tracking with four different solenoid models. IP is at $z=0 \mathrm{~m}$.
IP orbit produced by the solenoid radial and longitudinal fields.

A more systematic illustration of the effect of the solenoid and quadrupole overlap is presented in Figs. 7 and 8, where the solenoid field integral of the tiny detector is increased in two steps in order to add a small part of the field $(0.1$ and $0.5 \mathrm{~T} \mathrm{~m})$ on top of the FD quadrupoles. One can see that even a small amount of solenoid field inside the quadrupole significantly increases the coupling and beam size at IP.

Solenoid effects for different detector field models are summarized in Table I. It is clear that the major effect on the IP beam size comes from that part of the solenoid that overlaps with the final doublet. On the contrary, if the solenoid field does not enter the quadrupoles, there is a natural cancellation of the solenoid induced orbit at the IP and, to a major extent, suppression of the beam size 

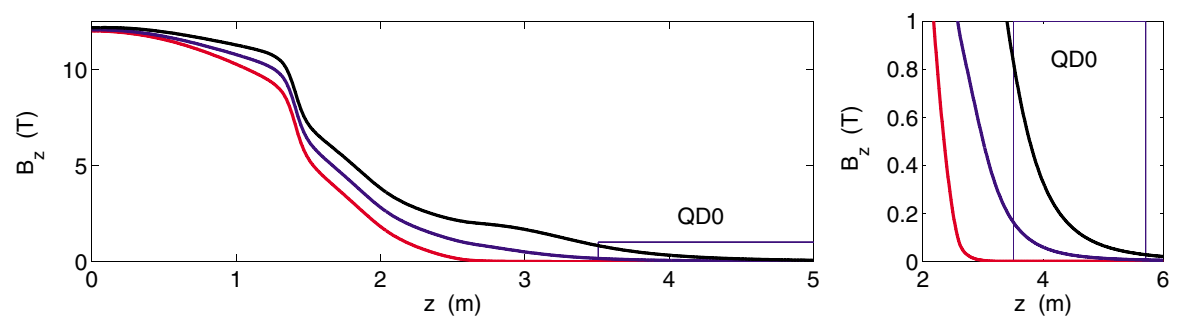

FIG. 7. (Color) Tiny detector with small additional field giving 0.1 or $0.5 \mathrm{Tm}$ integrated over FD (indicated as "Tiny $+\Delta_{1}$ " and "Tiny $+\Delta_{2}$ " in Table I). IP is at $z=0 \mathrm{~m}$.
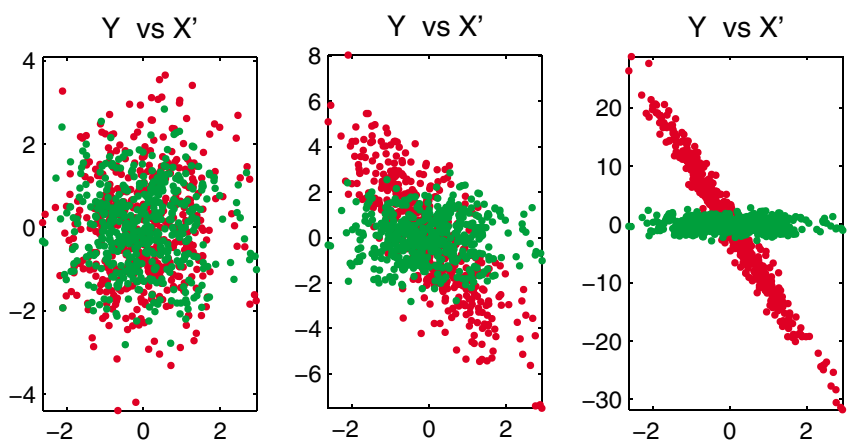

FIG. 8. (Color) Beam phase space at the IP in the tiny detector without (left) and with small additional field (middle, Tiny + $\Delta_{1}$; right, Tiny $+\Delta_{2}$ ).

growth. Table I also shows that a nonzero crossing angle does not significantly alter the uncompensated beam size.

In the IR designs for TESLA and NLC, the FD quadrupoles are placed inside the detector solenoid field. Earlier studies of solenoid compensation for these projects used skew quadrupole correction $[13,15,16]$. For both NLC and TESLA, a skew quadrupole placed at the final doublet removed most of coupling generated by the overlapped quadrupoles. By adding a second skew quadrupole or using a skew correction section and compensating the beam waist position, the IP beam size could be brought close to design. However, in this scheme the skew quadrupoles have to become stronger for lower beam energies.

Naturally, it is desirable to compensate the coupling effects of an overlapped quadrupole at its source, i.e., at the quadrupole, thus restoring the properties of a bare solenoid. In this paper, we discuss such a method of compensation in the linear collider IR using weak antisolenoids overlapping with the final doublet. An important feature of this scheme is that the weak antisolenoids do not need to compensate the entire field of the detector solenoid, but only the much smaller part which overlaps with the final doublet.

As will be shown below, compensation with weak antisolenoids is superior compared to skew quadrupole correction, and it is effective over the entire NLC energy range from 50 to $250 \mathrm{GeV} /$ beam. It is also insensitive to changes of the beam optics, and compatible with compensation of the vertical orbit angle at the IP [18].

\section{ANALYTIC DESCRIPTION OF EFFECTS OF SOLENOID OVERLAPPING WITH FINAL DOUBLET}

To investigate analytically the effect of the solenoid on the NLC beam, it is useful to consider a short test solenoid with field integral $B \ell=0.5 \mathrm{~T} \mathrm{~m}$, placed at various positions near the IP, instead of the full detector solenoid. The overall effect of a realistic solenoid field can then be evaluated by integration.

Two cases are discussed: (1) the test solenoid is coaxial with the detector and (2) the test solenoid is coaxial with the beam, analogous to the possible configurations of the

TABLE I. Uncompensated solenoid effects on the $250 \mathrm{GeV}$ beam for different NLC detector models. Here $B \ell$ is the full field integral over half the detector, and $B \ell_{\mathrm{FD}}$ is its fraction over the final doublet, i.e., for $z>3.51 \mathrm{~m}$. The correlation terms $\left\langle y x^{\prime}\right\rangle$ and $\langle y E\rangle$ are normalized to the nominal rms beam sizes.

\begin{tabular}{lccccccc}
\hline \hline Detector & $B \ell(\mathrm{Tm})$ & $B \ell_{\mathrm{FD}}(\mathrm{Tm})$ & $y_{\mathrm{IP}}(\mu \mathrm{m})$ & $\sigma_{x} / \sigma_{x 0}$ & $\sigma_{y} / \sigma_{y 0}$ & $\left\langle y x^{\prime}\right\rangle$ & $\langle y E\rangle$ \\
\hline SiD & 16.7 & 1.7 & -18.5 & 1.1 & 31.5 & -32.8 & -0.33 \\
SiD, 0-xing & 16.7 & 1.7 & 0 & 1.1 & 43.6 & -32.7 & -28.4 \\
LD & 14.4 & 4.4 & -74.5 & 2.2 & 149.2 & -153.4 & -18.0 \\
LD, 0-xing & 14.4 & 4.4 & 0 & 2.1 & 187.1 & -141.1 & -122.9 \\
Tiny & 18.0 & 0 & 0 & 1.0 & 1.31 & 0.0 & 0.0 \\
Tiny $+\Delta_{1}$ & 20.2 & 0.1 & -1.05 & 1.0 & 2.34 & -2.00 & -0.15 \\
Tiny $+\Delta_{2}$ & 22.1 & 0.5 & -5.22 & 1.03 & 10.5 & -10.51 & -0.98 \\
\hline \hline
\end{tabular}


weak compensating antisolenoid discussed in Sec. V that can be built into the detector or wound on a final quadrupole. In both cases, the incoming beam travels at an angle to the detector axis equal to half of the NLC crossing angle $\theta_{c}$. The difference between the two cases is the radial field seen by the beam at the entrance to the test solenoid. In the first case, this field is not zero since the incoming beam is horizontally offset with respect to the solenoid axis. In the second case, there is no radial field on the beam axis.

The derivations presented in this section are performed in the approximation of a weak solenoid and assumption that solenoid transverse focusing can be neglected, in particular, that the unperturbed design Twiss functions of BDS can be used, and the effects of the solenoid focusing on the orbit are small. The validity of this assumption will be discussed later.

Below, we will first analyze the beam orbit caused by the solenoid, which is nonzero only for the case of the test solenoid placed on the detector axis, and then investigate the beam correlations at the IP. This will lead us to the conditions for cancellation of the solenoid effects and to the concept of weak compensating antisolenoids.

Let us consider how the test solenoid placed on the detector axis affects the beam orbit. As follows from Eq. (9), a thin solenoid located at distance $Z_{s}$ from the IP and traversed at horizontal radius $-Z_{s} \theta_{c}$ and angle $\theta_{c}$ will cause the vertical orbit and angle to change by

$$
\Delta y=\frac{B \ell Z_{s} \theta_{c}}{2 B \rho} \quad \text { and } \quad \Delta y^{\prime}=-\frac{B \ell \theta_{c}}{2 B \rho} .
$$

Clearly, if there are no focusing elements between the solenoid and the IP, then the vertical offset at the IP will exactly cancel since $y_{\mathrm{IP}}=\Delta y+Z_{s} \Delta y^{\prime}=0$. If there is a quadrupole field after the solenoid, it focuses the orbit and breaks the cancellation. Note that the IP orbit angle produced by the solenoid does not cancel.

An example of the orbit created by the test solenoid located at $Z_{s}=10 \mathrm{~m}$ is shown in Fig. 9. Here the orbit obtained by tracking in DIMAD is compared with an analytic calculation integrating the horizontal field $B_{x}$ of the solenoid and FD quadrupoles (including the additional case of a very thin solenoid with $\ell \rightarrow 0$ ):

$$
\frac{d^{2} y}{d z^{2}}=\frac{B_{x}}{B \rho}+\frac{G(z) y}{B \rho},
$$

where $G(z)$ is the gradient of the FD quadrupoles. One can see that the thin solenoid approximation is in a good agreement with the calculation of the exact model using analytic and tracking methods. In DIMAD tracking, the FD region was modeled by a sequence of short, typically $1 \mathrm{~cm}$ long slices containing all the solenoid, dipole, quadrupole, sextupole, and octupole fields, using the realistic solenoid field map and the design BDS optics.

Using the formalism of beam transport matrices, the orbit at the IP can be expressed as
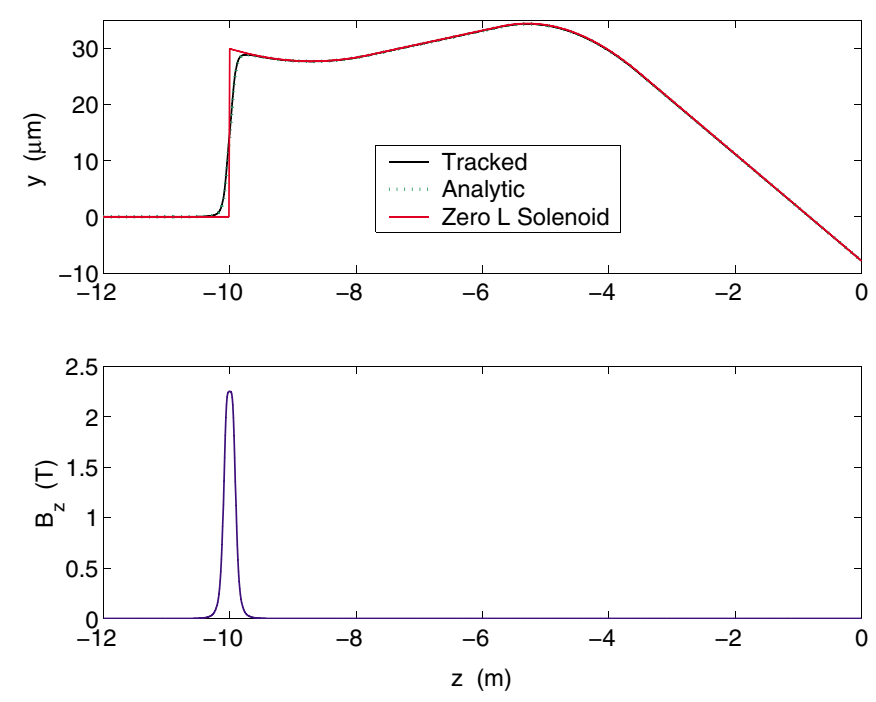

FIG. 9. (Color) Orbits with a short test solenoid of $B \ell=0.5 \mathrm{~T} \mathrm{~m}$ placed on the detector axis at $10 \mathrm{~m}$ from the NLC IP. Orbits are obtained using particle tracking and analytical integration of the field for the exact model, and with a zero length approximation of the solenoid. IP is at $z=0 \mathrm{~m}$.

$$
y_{\mathrm{IP}}=\Delta y m_{33}+\Delta y^{\prime} m_{34}, \quad y_{\mathrm{IP}}^{\prime}=\Delta y m_{43}+\Delta y^{\prime} m_{44} .
$$

Here $m_{i j}$ are elements of the transport matrix $M$ from the test solenoid to the IP. Substituting the well-known Twiss expressions for the matrix terms, the formula for $y_{\mathrm{IP}}$ can be written as

$$
y_{\mathrm{IP}}=\frac{B \ell \theta_{c}}{2 B \rho} \sqrt{\beta_{y 0} / \beta_{y}}\left(Z_{s} C_{Y}+Z_{s} \alpha_{y} S_{Y}-\beta_{y} S_{Y}\right),
$$

where $C_{X}=\cos \left(\mu_{x}\right), S_{X}=\sin \left(\mu_{x}\right), C_{Y}=\cos \left(\mu_{y}\right)$, and $S_{Y}=\sin \left(\mu_{y}\right)$ are functions of the horizontal and vertical phase advance $\mu_{x, y}$ between the test solenoid and the IP. $\beta$ and $\alpha$ are the Twiss parameters at the location of the test solenoid, and $\beta_{0}$ and $\alpha_{0}=0$ are the Twiss parameters at IP, for the corresponding planes. Taking into account the analytic expressions for $\beta, \alpha$, and $\mu$, one can verify that in the final drift before the IP

$$
Z_{s} C_{Y}+Z_{s} \alpha_{y} S_{Y}-\beta_{y} S_{Y}=0,
$$

and hence $y_{\mathrm{IP}}$ is exactly cancelled if there is only a drift between the test solenoid and the IP. Note that in Eq. (14) the terms which mutually cancel are very large, of the order of $L_{*}^{2} / \beta_{y 0}$, where $L_{*}$ is the length of the final drift. If the test solenoid overlaps the FD quadrupoles, the sine terms become dominant, and the maximum offset at the IP can be estimated roughly as

$$
y_{\mathrm{IP}} \approx \frac{B \ell \theta_{c}}{B \rho} L_{*} .
$$

The effect of the test solenoid on the beam offset at the IP is shown in Fig. 10 (lower plot), where one can see the 

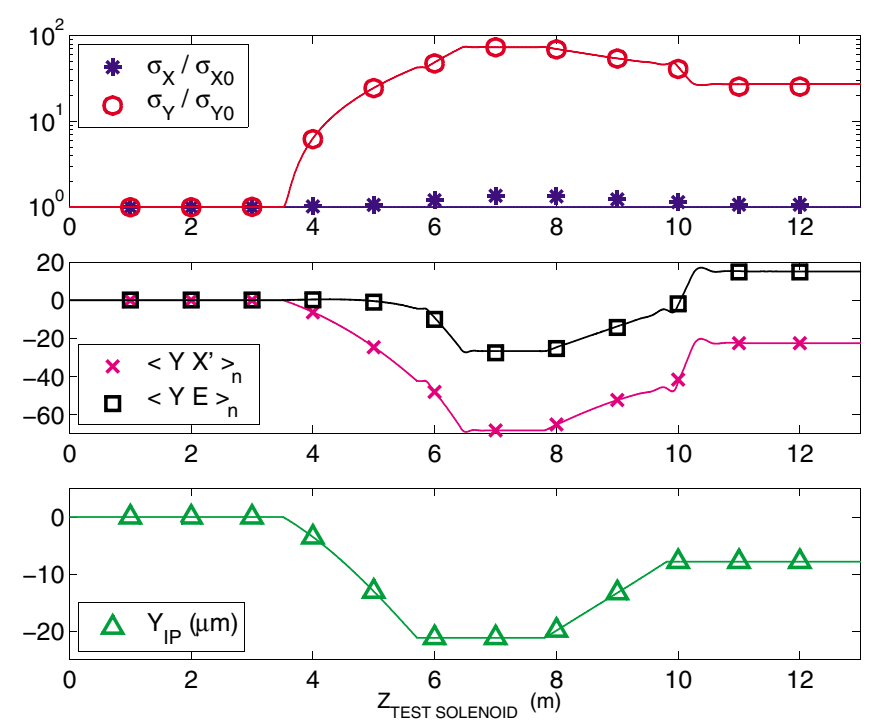

FIG. 10. (Color) Beam size, major correlations, and $Y$ position at the IP for the short test solenoid placed on the detector axis at distance $z$ from IP. Symbols: tracking; solid line: analytic solutions. IP is at $z=0 \mathrm{~m}$.

perfect orbit cancellation when the test solenoid is in the last drift and good agreement between tracking and analytical results obtained using Eq. (13). Similar calculation for the test solenoid placed on the beam axis is shown in Fig. 11.

Now let us consider the effect of the solenoid on the dominant IP aberration terms - the coupling $\left\langle y x^{\prime}\right\rangle$ and vertical dispersion $\langle y E\rangle$. Figures 10 and 11 show these terms and the beam size growth at the IP as a function of the test solenoid position, where the tracking results are
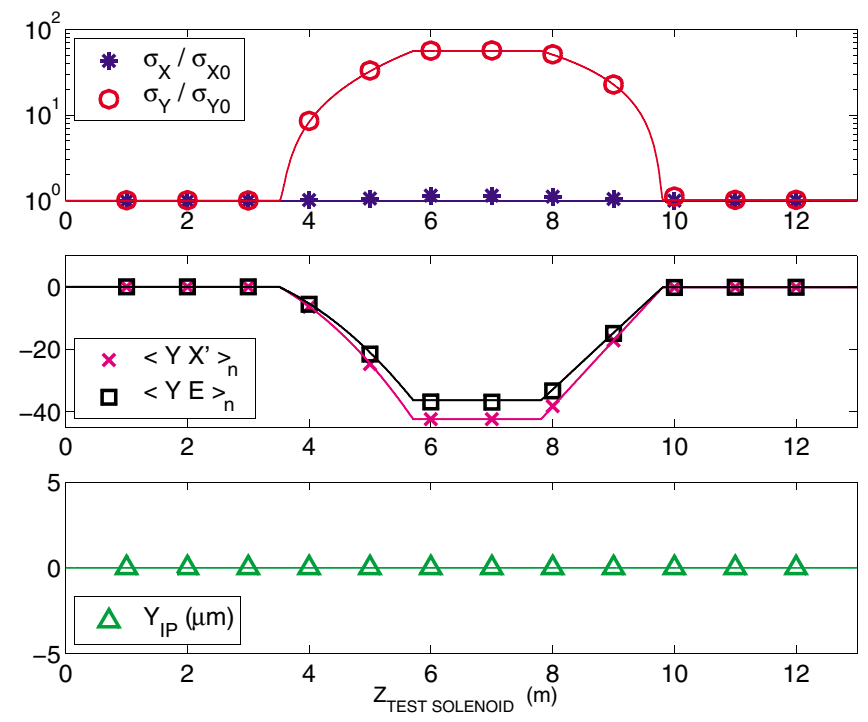

FIG. 11. (Color) Beam size, major correlations, and $Y$ position at the IP for the short test solenoid placed on the beam axis at distance $z$ from IP. Symbols: tracking; solid line: analytic solutions. IP is at $z=0 \mathrm{~m}$. compared with analytical values given by the expressions derived below.

If the test solenoid is placed on the beam axis, the projected coupling term $\left\langle y x^{\prime}\right\rangle$ does not depend on the crossing angle and is given by (normalized to the nominal rms beam sizes):

$$
\left\langle y x^{\prime}\right\rangle_{\theta=0}=\frac{\sigma_{x p 0}}{\sigma_{y 0}} \frac{B \ell}{2 B \rho}\left(m_{12} m_{33}-m_{11} m_{34}\right) .
$$

This equation is derived by taking into account that the solenoid matrix $M_{S}$ projected to IP [10] is

$$
M_{S \text { proj }}=M \quad M_{S} \quad M^{-1} .
$$

Expanding Eq. (16) in terms of Twiss parameters, we obtain

$$
\begin{aligned}
\left\langle y x^{\prime}\right\rangle_{\theta=0}= & \frac{\sigma_{x p 0}}{\sigma_{y 0}} \frac{B \ell}{2 B \rho} \frac{\sqrt{\beta_{x 0} \beta_{y 0}}}{\sqrt{\beta_{x} \beta_{y}}}\left(\alpha_{y} \beta_{x} S_{X} S_{Y}+\beta_{x} S_{X} C_{Y}\right. \\
& \left.-\beta_{y} S_{Y} C_{X}-\alpha_{x} \beta_{y} S_{X} S_{Y}\right) .
\end{aligned}
$$

The terms in the parentheses are very large, of the order of $L_{*}^{3} /\left(\beta_{x 0} \beta_{y 0}\right)$; however, they again cancel exactly when the test solenoid is in the last drift. The latter is easy to see applying $m_{11}=m_{33}=1$ and $m_{12}=m_{34}=Z_{s}$ in Eq. (16), or using Eq. (14). Another case of exact cancellation is the so-called "parallel-to-point" transformation, where $m_{11}=$ $m_{33}=0$, which roughly represents the matrix from the drift prior to the final doublet where the beam is almost parallel. In general, cancellation of $\left\langle y x^{\prime}\right\rangle$ in Eq. (16) occurs for a symmetric $x$ and $y$ transformation with $m_{11}=m_{33}$ and $m_{12}=m_{34}$. This can also be concluded from Eqs. (5) and (6) which show that a symmetric $x-y$ matrix commutes with the solenoid rotation matrix, hence does not change the solenoid coupling.

Obviously, when the test solenoid is inside the final doublet, the opposite quadrupole focusing in two planes breaks the $x$ and $y$ symmetry and, hence, the cancellation of $\left\langle y x^{\prime}\right\rangle$. Thus, one can conclude that the physical reason for the breakdown of the cancellation is that the FD quadrupoles affect two planes differently, i.e., defocus in one plane and focus in another.

The maximal effect of this coupling term, with the test solenoid in the FD, can be estimated as

$$
\left\langle y x^{\prime}\right\rangle_{\theta=0} \approx \frac{\sigma_{x p 0}}{\sigma_{y 0}} \frac{B \ell}{B \rho} L_{*} .
$$

We can see in Fig. 11 that agreement between tracking and the analytical formula is very good. It is also very interesting to note, from this figure, that there is a very good cancellation also for the case when the test solenoid is placed after the final doublet. As we just discussed above, this happens because the beam after the final doublet is almost parallel in both planes. Equation (18) shows that in this case the cancellation is only approximate, since the 
effective image points upstream of the FD for the $x$ and $y$ planes usually do not occur at the same location.

For the test solenoid placed on the beam axis, the vertical dispersion at the IP $\langle y E\rangle$ arises from the solenoid coupling of the horizontal dispersion into the vertical plane:

$$
\langle y E\rangle_{\theta=0}=-\frac{\sigma_{E}}{\sigma_{y 0}} \frac{B \ell}{2 B \rho}\left(m_{33} \eta_{x}+m_{34} \eta_{x}^{\prime}\right),
$$

where $\eta_{x}$ and $\eta_{x}^{\prime}$ are the horizontal dispersion and its derivative at the location of the test solenoid. Expanding, we obtain

$$
\begin{aligned}
\langle y E\rangle_{\theta=0}= & -\frac{\sigma_{E}}{\sigma_{y 0}} \frac{B \ell}{2 B \rho}\left[\sqrt{\beta_{y 0} / \beta_{y}}\left(C_{Y}+\alpha_{y} S_{Y}\right) \eta_{x}\right. \\
& \left.+\sqrt{\beta_{y 0} \beta_{y}} S_{Y} \eta_{x}^{\prime}\right] .
\end{aligned}
$$

For the case where the angular horizontal dispersion is nominally nonzero at the IP, and the dispersion itself is zero, Eq. (20) can be rewritten as

$$
\langle y E\rangle_{\theta=0}=\frac{\sigma_{E}}{\sigma_{y 0}} \frac{B \ell}{2 B \rho} \eta_{x 0}^{\prime}\left(m_{12} m_{33}-m_{11} m_{34}\right),
$$

which has exactly the same dependence as the coupling term in Eq. (16). This means that both major correlations can be corrected simultaneously by the weak antisolenoid discussed in Sec. V.

Figure 11 shows that agreement between the simulations and these analytical expressions is good. Similar to Eq. (19), the estimate for the maximal effect on the vertical dispersion term can be written as

$$
\langle y E\rangle_{\theta=0} \approx \frac{\sigma_{E}}{\sigma_{y 0}} \frac{B \ell}{B \rho} \eta_{x 0}^{\prime} L_{*} .
$$

Let us now derive the coupling and dispersion terms for the case when the test solenoid is placed on the detector axis. This case must include the kick on the orbit $\Delta y$ and $\Delta y^{\prime}$ at the location of the test solenoid as given by Eq. (10). Since these kicks are large, the second order transfer matrix $T$ (its elements are $t_{i j k}$ ) from the test solenoid to the IP must also be taken into account.

Correspondingly, the $\left\langle y x^{\prime}\right\rangle$ coupling will be given by

$$
\begin{aligned}
\left\langle y x^{\prime}\right\rangle= & \left\langle y x^{\prime}\right\rangle_{\theta=0}+\frac{\sigma_{x p 0}}{\sigma_{y 0}}\left[-\left(t_{331} \Delta y+t_{341} \Delta y^{\prime}\right) m_{12}\right. \\
& \left.+\left(t_{332} \Delta y+t_{342} \Delta y^{\prime}\right) m_{11}\right]
\end{aligned}
$$

where the additional term is proportional to $\theta_{c}$.

Similarly, the vertical dispersion $\langle y E\rangle$ will have three additional terms: (a) the term due to the energy dependence of the kicks $\Delta y$ and $\Delta y^{\prime}$ :

$$
\begin{aligned}
\delta\langle y E\rangle & =\frac{\sigma_{E}}{\sigma_{y 0}}\left(-m_{33} \Delta y-m_{34} \Delta y^{\prime}\right) \\
& =\frac{\sigma_{E}}{\sigma_{y 0}} \frac{B \ell}{2 B \rho}\left(-m_{33} Z_{s}+m_{34}\right) \theta_{c} ;
\end{aligned}
$$

(b) the term due to the vertical orbit offset in the quadrupoles of the final doublet, which can be obtained by integration of the known orbit or, equivalently, by using the second order matrices:

$$
\delta\langle y E\rangle=\frac{\sigma_{E}}{\sigma_{y 0}}\left(t_{336} \Delta y+t_{346} \Delta y^{\prime}\right) ;
$$

and finally (c) the term driven by the horizontal dispersion $\eta_{x}$ and $\eta_{x}^{\prime}$ and orbit kick $\Delta y$ and $\Delta y^{\prime}$ via the second order matrix $T$ :

$$
\begin{aligned}
\delta\langle y E\rangle= & \frac{\sigma_{E}}{\sigma_{y 0}}\left[\left(t_{331} \Delta y+t_{341} \Delta y^{\prime}\right) \eta_{x}\right. \\
& \left.+\left(t_{332} \Delta y+t_{342} \Delta y^{\prime}\right) \eta_{x}^{\prime}\right] .
\end{aligned}
$$

Figure 10 shows that the analytical results agree perfectly with tracking. Figure 12 shows that contributions to the total effect can combine in a more or less favorable manner, depending on the sign of the dispersion with respect to the crossing angle, which is defined by the geometry of the beam delivery and the location of the detector. In particu-
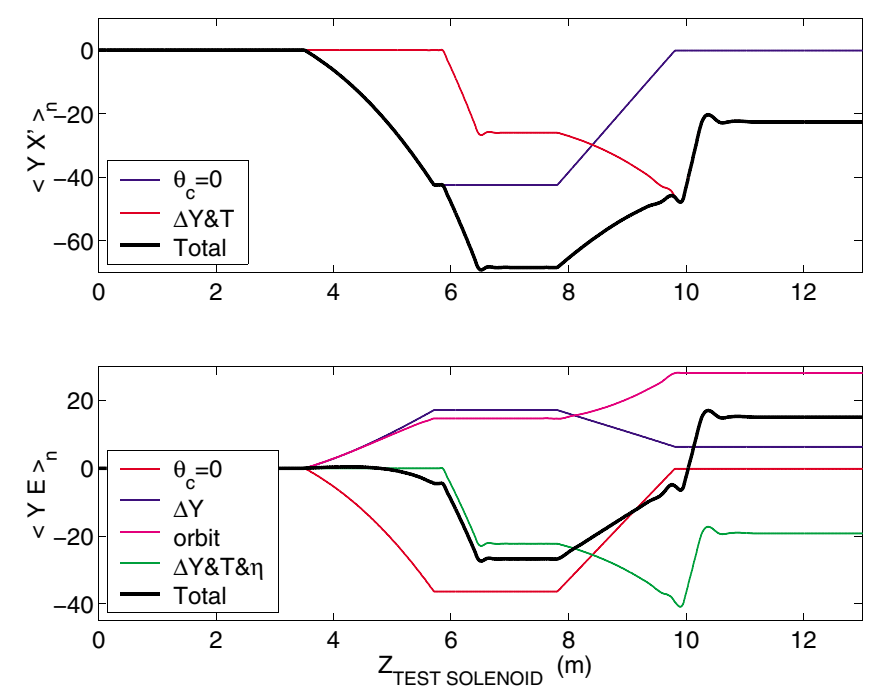

FIG. 12. (Color) Contributions of different terms to the coupling $\left\langle y x^{\prime}\right\rangle$ and vertical dispersion $\langle y E\rangle$ for the test solenoid placed on the detector axis, based on analytic calculation. Coupling (top plot) includes a term independent of crossing angle (blue line) and a term from the orbit kicks and the second order matrix (red line). Dispersion (bottom plot) includes contribution from the angle independent coupling (red line), and three additional terms due to the energy dependence of the kicks (blue line), to the vertical offset in the quadrupoles (magenta line), and to the second order matrix, horizontal dispersion and orbit kick (green line). IP is at $z=0 \mathrm{~m}$. 
lar, the reason that the vertical dispersion is smaller with the nominal crossing angle, as seen in Table I, is the partial compensation of the horizontal dispersion and crossing angle terms.

For the case of zero crossing angle, it was shown above that a weak antisolenoid overlapping the FD can compensate both the $\left\langle y x^{\prime}\right\rangle$ coupling and $\langle y E\rangle$ dispersion simultaneously. In this case, the condition for the combined detector solenoid and antisolenoid field to restore the solenoid cancellation at the IP is to have

$$
\int_{-\infty}^{0} \frac{B_{z}}{B \rho}\left(m_{12} m_{33}-m_{11} m_{34}\right) d z=0
$$

or, equivalently,

$$
\begin{aligned}
\int_{-\infty}^{0} \frac{B_{z}}{B \rho} \frac{1}{\sqrt{\beta_{x} \beta_{y}}}\left(\alpha_{y} \beta_{x} S_{X} S_{Y}+\beta_{x} S_{X} C_{Y}-\right. \\
\left.\beta_{y} S_{Y} C_{X}-\alpha_{x} \beta_{y} S_{X} S_{Y}\right) d z=0 .
\end{aligned}
$$

Note that the validity of the above integration for the combined field of the detector solenoid and antisolenoids follows from the use of the projection method. In this approach, the total solenoid field can be replaced by a sequence of short solenoid slices. Starting from the nearest to IP slice, they all can be effectively projected to IP with the coupling terms of the same form as derived earlier for the short test solenoid. With the assumption of negligible focusing from the solenoid, the projected coupling matrix terms at IP from all slices will linearly add, thus validating the integration.

It is also clear that the conditions for cancellation of the solenoid effects do not depend on the optics parameters at the IP. For example, changing the IP $\beta$ function or angular dispersion $\eta_{0}$ would not affect the cancellation because they do not change the final doublet strength, which is defined to a major extent just by the geometry. Therefore, all matrix elements remain the same, and Eq. (28) remains valid independent of the IP beam parameters.

In the case with a crossing angle, it may be possible to express the cancellation conditions in a similar manner. In this case, however, the analytical approach would require derivation of the behavior of all the linear and second order optics functions along the longitudinal coordinate in the IR of the final focus, which goes well beyond the scope of the paper. We therefore will resort to computer simulations and demonstrate in the next section that the weak antisolenoid compensation can be achieved for the nonzero crossing angle and different detector models considered in this paper.

Finally, we include formulas for the two other beam correlation terms which do not cancel even if the solenoid does not overlap the final doublet:

$$
\langle y x\rangle=\frac{\sigma_{x 0}}{\sigma_{y 0}} \int_{-\infty}^{0} \frac{B_{z}}{2 B \rho}\left(m_{21} m_{34}-m_{22} m_{33}\right) d z
$$

and

$$
\left\langle y y^{\prime}\right\rangle=\frac{\sigma_{y^{\prime} 0}}{\sigma_{y 0}} \int_{-\infty}^{0}\left(\frac{B_{z}}{2 B \rho} m_{34}\right)^{2} d z
$$

where the former corresponds to beam rotation, and the latter is the solenoid focusing proportional to the square of the field. These terms do not depend on $\theta_{c}$, and they are usually much smaller than the other beam distortions considered above.

As noted above, the solenoid focusing was neglected in the presented consideration. Taking this focusing into account, it is easy to estimate that a short test solenoid placed in the final drift would result in the following residual offset at the IP:

$$
\Delta y_{\mathrm{IP}} \approx-\left(\frac{B \ell}{2 B \rho}\right)^{3} \frac{\theta_{c} Z_{s}^{2}}{\ell},
$$

for $Z_{s} \gg \ell$. This would give about $0.024 \mathrm{~nm}$ if the above test solenoid is located at $Z_{s}=3 \mathrm{~m}$. For the solenoid field that fills the final drift entirely, the estimate is

$$
\Delta y_{\mathrm{IP}} \approx-\left(\frac{B \ell}{2 B \rho}\right)^{3} \frac{\theta_{c} L_{*}}{3},
$$

yielding about $10 \mathrm{~nm}$ for a typical detector model at the nominal parameters, which is sufficiently small and does not affect the validity of formulas presented in this section.

\section{COMPENSATION OF IP BEAM SIZE USING WEAK ANTISOLENOIDS}

In this section, the method of the weak antisolenoids is illustrated on the example of compensation of the $\mathrm{SiD}$ solenoid. Performance of the method is then investigated as a function of beam energy, and compared with the compensation using skew quadrupoles. Compensation of the LD solenoid was performed in a similar way and is described in detail in [19]. This study showed that the weak antisolenoids provide good compensation for the sufficiently different SiD and LD detector solenoid models. Based on these results and flexibility of the method, we conclude that this compensation can be applied to any realistic model of detector solenoid field with the overlapped quadrupoles.

The fields of the SiD with an optimized weak antisolenoid are shown in Fig. 13. The antisolenoid is placed on the detector axis, and its field is approximated by

$$
B_{z \text { antisol }}=\frac{B_{z 0}}{1+\left[\left(z-z_{0}\right) / z_{w}\right]^{4}},
$$

where the location $z_{0}=4.14 \mathrm{~m}$, width $z_{w}=0.5 \mathrm{~m}$, and strength $B_{z 0}=-1.56 \mathrm{~T}$ are optimized to cancel the beam offset at the IP and the two major beam distortions. The integral strength of this solenoid is $1.74 \mathrm{~T} \mathrm{~m}$. The particular functional dependence of this approximation is irrelevant 

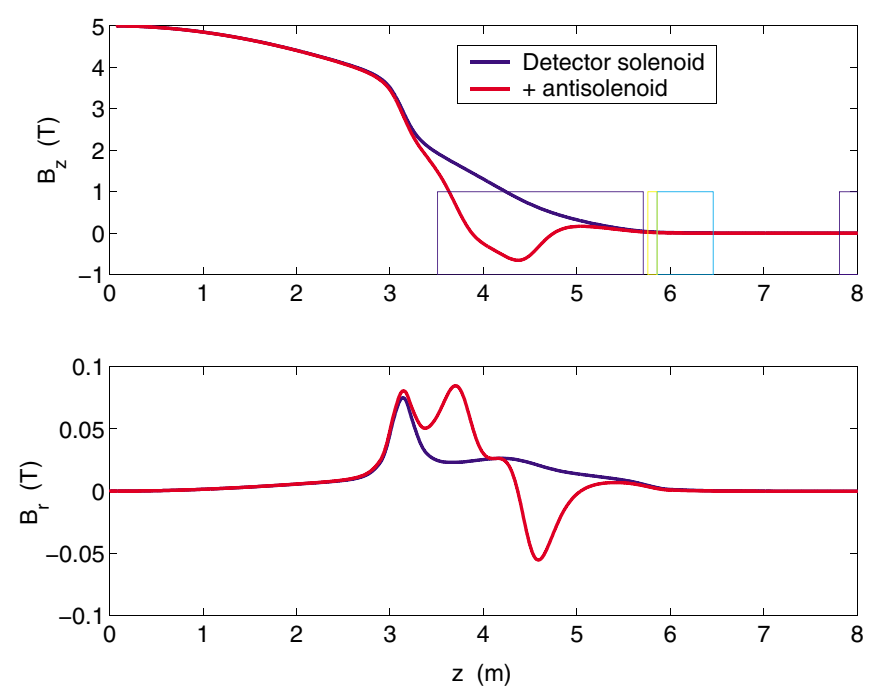

FIG. 13. (Color) Fields in $\mathrm{SiD}$ with and without the optimized weak antisolenoid. IP is at $z=0 \mathrm{~m}$.

because the parameters can be easily reoptimized for calculated fields of realistic coils.

Orbits through the $\mathrm{SiD}$ with this antisolenoid are shown in Fig. 14. The IP vertical orbit offset is now canceled. ${ }^{1}$ The beam phase space in $\mathrm{SiD}$ with this antisolenoid but without any other correcting knobs is shown in Fig. 15. The beam size is compensated to within $30 \%$. Comparing with the bare SiD results given in Fig. 5 and Table I, it appears that this weak antisolenoid has compensated $99 \%$ of the effect.

The remaining deviation of the beam size from nominal is easy to correct with linear tuning knobs. These include BDS sextupole displacement knobs and skew quadrupole knobs in the skew correction section of the BDS, which allow orthogonal correction of the $\langle y E\rangle,\left\langle y y^{\prime}\right\rangle,\langle y x\rangle$, and $\left\langle y x^{\prime}\right\rangle$ terms, and similar terms in the horizontal plane, if necessary. The beam phase space in SiD with the antisolenoid, and with additional optimal linear knobs, is shown in Fig. 16. One can see that the beam size is compensated to within $0.3 \%$ of nominal, which is within the resolution.

We have seen earlier in Table I and Sec. IV that with the bare $\mathrm{SiD}$ or $\mathrm{LD}$ solenoids, and with a positive crossing angle, the vertical dispersion term $\langle y E\rangle$ is rather small because the crossing angle term and the horizontal angular dispersion term approximately cancel each other. For a negative crossing angle, the $\langle y E\rangle$ term is doubled. However, this peculiar dependence on the crossing angle

\footnotetext{
${ }^{1}$ The small nonzero horizontal offset at the IP can be easily corrected, and the vertical angle is of no concern for luminosity, since in the $e^{+} e^{-}$collider the orbits will be antisymmetrical, and the beams will still collide head-on. For the $e^{-} e^{-}$version of the collider, or if more careful preservation of the beam polarization is needed, the vertical angle at the IP can also be locally compensated using the so-called detector integrated dipole (DID) corrector, as suggested in [18], which is compatible with the beam size compensation using weak antisolenoids.
}
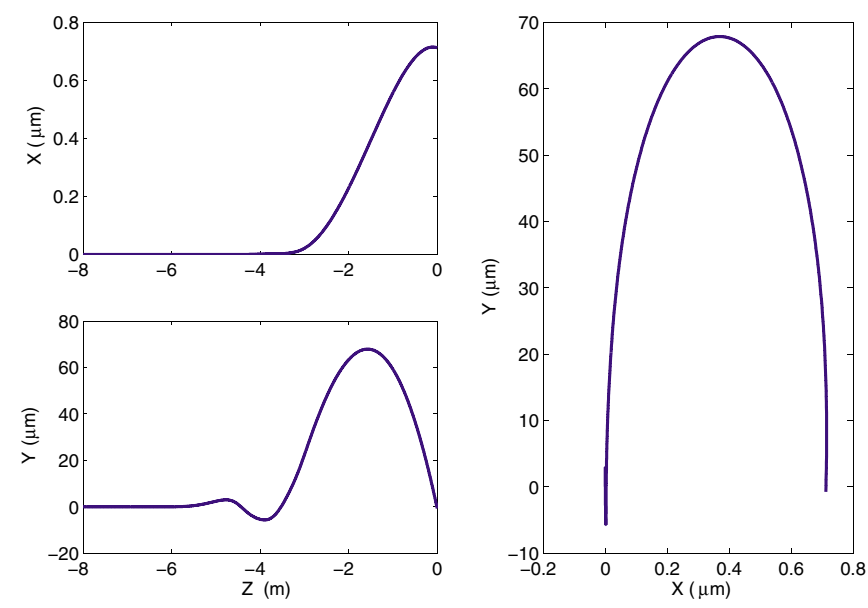

FIG. 14. (Color) Orbits with SiD fields compensated by a weak antisolenoid. IP is at $z=0 \mathrm{~m}$.

mostly disappears when the antisolenoid is used. Figure 17 shows the dependence of the beam IP position and major beam distortions $\left\langle y x^{\prime}\right\rangle$ and $\langle y E\rangle$ on the crossing angle, with and without the weak antisolenoid. The antisolenoid compensates the vertical IP position, coupling, and vertical dispersion, and also eliminates most of the dependence on the crossing angle.

Agreement between analytical and tracking results shown in Fig. 17 is acceptable. A small difference of about 0.1 in the vertical dispersion term and several tens of nanometers in the vertical orbit is explained by the effect of the solenoid focusing, which was neglected in our analytic derivations. One can see that the assumption of the negligible solenoid focusing is indeed justified for our case, since these differences are small.

Regarding the technical implementation of the weak antisolenoid, it is essential that this antisolenoid is aligned on the detector axis, so it should be built as an integral part of the SiD detector. The antisolenoid should have at least two coils, separated longitudinally and individually powered, in order to provide fine adjustment of both the amplitude and longitudinal position of the antisolenoid field. It should be compact, to minimize interference and space taken from the detector, and should be able to withstand the longitudinal forces acting on the antisolenoid due to the main solenoid field (these forces prohibit the use of an antisolenoid wound on QD0 quadrupole and placed on the beam axis). The necessary accuracy of the antisolenoid parameters are rather relaxed. In particular, in the $\mathrm{SiD}$ case the following variations of the antisolenoid parameters would cause the beam correlations to change by about $30 \%$ (thus beam size to change by about $10 \%$ ): $\Delta z_{0} \approx$ $6 \mathrm{~mm}, \Delta z_{w} \approx 40 \mathrm{~mm}, \Delta B_{z 0} / B_{z 0} \approx 1 \%$.

The use of antisolenoids to shielding the final quadrupole was considered by the GLC project [20], but their purpose was to protect the iron electromagnetic quadrupole from saturation in the solenoid field and not for beam size compensation. The design of this solenoid can be modified 

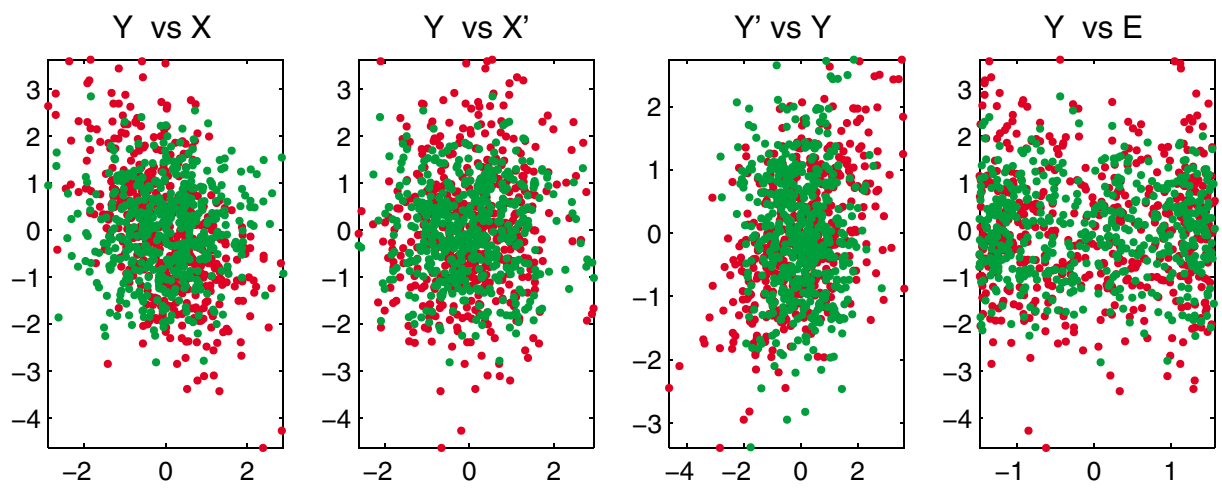

FIG. 15. (Color) Beam phase space in $\mathrm{SiD}$ with an antisolenoid but without any correction knobs. The beam size is $\sigma_{x} / \sigma_{x 0}=1.00$, $\sigma_{y} / \sigma_{y 0}=1.29$.
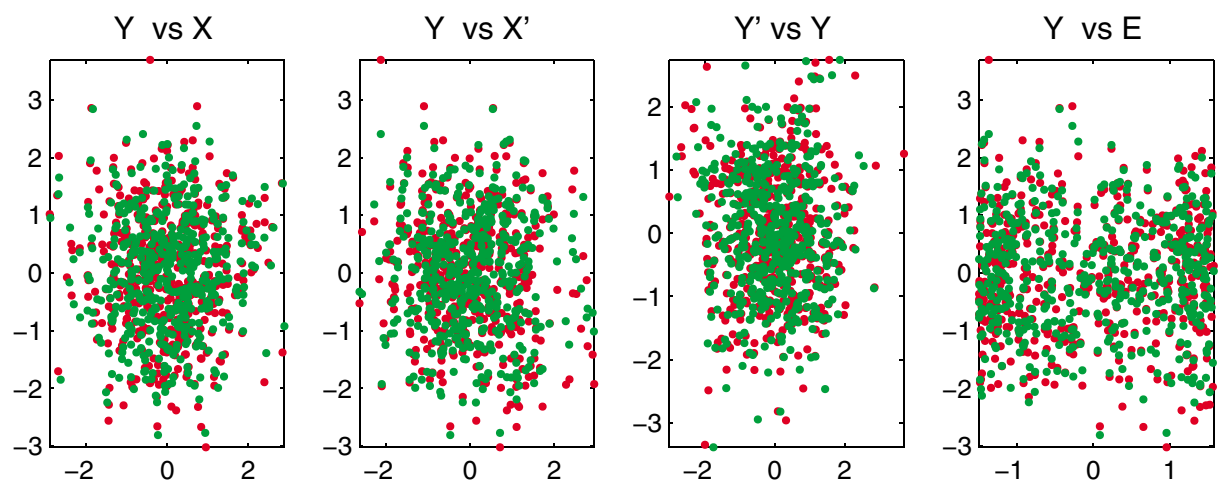

FIG. 16. (Color) Beam phase space in $\mathrm{SiD}$ with an antisolenoid and with additional correction knobs. The beam size is $\sigma_{x} / \sigma_{x 0}=$ $1.00, \sigma_{y} / \sigma_{y 0}=1.003$.

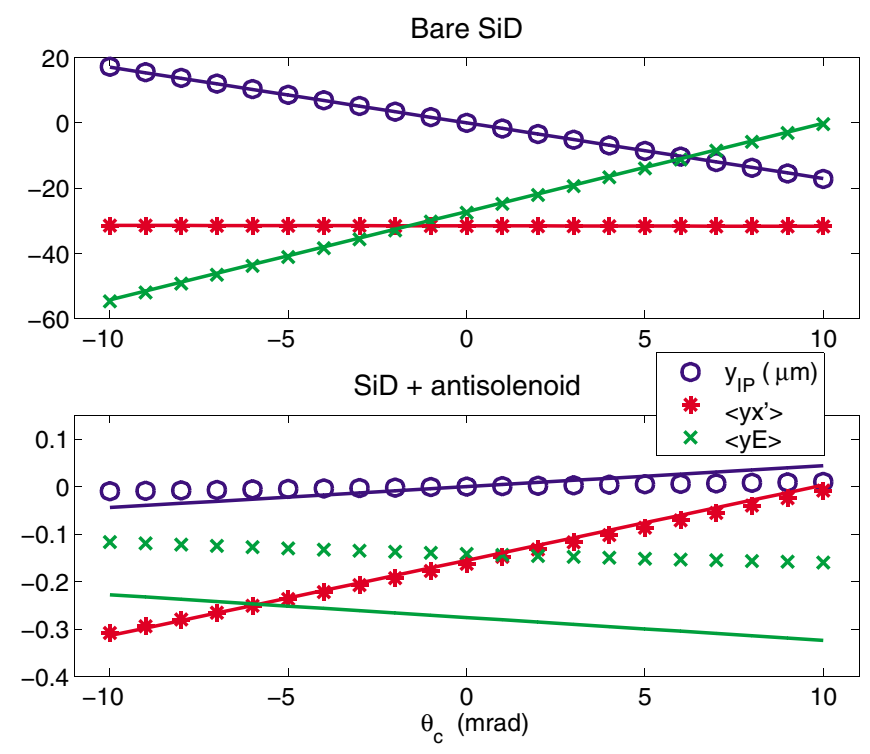

FIG. 17. (Color) Vertical orbit at the IP, and beam distortions $\left\langle y x^{\prime}\right\rangle$ and $\langle y E\rangle$ in $\mathrm{SiD}$ without (top plot) and with antisolenoid (bottom plot) as a function of crossing angle. Symbols: tracking; lines: analytical results. to also provide beam size compensation. In this case, the iron of the quadrupole would also shield part of the solenoid field, helping the compensation, but any remaining field beyond the first quadrupole in the FD would also need to be compensated.

The antisolenoids optimized for the LD case are described in [19]. Here the field extends much farther into the FD, and it was found that a single antisolenoid solution may not be practical, since the antisolenoid location extends into the detector tracking region by about a meter. A more practical solution for LD consists of two antisolenoids, the main one is located on the detector axis in the LD yoke region (thus do not interfere with detector operation) and overlaps with part of QD0 $\left(z_{0}=5.4 \mathrm{~m}, z_{w}=0.6 \mathrm{~m}\right.$, $B_{z 0}=-1.7 \mathrm{~T}$, the integral strength $2.3 \mathrm{~T} \mathrm{~m}$ ), and the second one is on the beam axis, overlapping with QF1 $\left(z_{0}=\right.$ $8.8 \mathrm{~m}, z_{w}=0.8 \mathrm{~m}, B_{z 0}=0.04 \mathrm{~T}$, the integral strength $0.07 \mathrm{~T} \mathrm{~m})$. This second solenoid could actually be wound on QF1 (provided QF1 is not an iron quadrupole) since the forces from the main solenoid are already very small. In spite of the fact that this second antisolenoid is very weak, it gives an additional flexibility to correct all the beam correlations and to move the main antisolenoid outside of 
the LD central part. Only with the two antisolenoids the beam size is compensated within $23 \%$ of nominal. Thus the weak antisolenoids compensated $99.8 \%$ of the effect. With the antisolenoids and with additional linear knobs the beam size is compensated within $0.9 \%$ of nominal.

Compensation with weak antisolenoids is very effective. The integrated strength of these antisolenoids is only $10 \%-$ $15 \%$ of the $\mathrm{SiD}$ or LD integrated strength, so they can be made compact to minimize interference with the detector, yet they cancel more than $99 \%$ of the effect of the detector solenoid. The remaining beam distortions are sufficiently small to be easily corrected with the linear tuning knobs of the BDS.

To maximize physics capabilities, the detector should operate with the same solenoid field for any beam energy in the range of the collider. For the first stage of NLC operation, this spans from 50 to $350 \mathrm{GeV}$, with a nominal beam energy of $250 \mathrm{GeV}$. Thus, it is important to characterize the performance of the beam size compensation over the full range of energies. As the beam energy decreases, the solenoid effect on the beam size increases at least linearly, and for certain terms quadratically [such as $\left\langle y y^{\prime}\right\rangle$, see Eq. (31)].

The effect of the SiD solenoid on the IP beam size as a function of energy is shown in Fig. 18 (top plot). The beam size at $50 \mathrm{GeV}$ increases to more than a hundred times the nominal size (the latter depends on energy as $1 / \sqrt{E}$ ). The dominant beam distortion is $\left\langle y x^{\prime}\right\rangle$ coupling, and the second largest term at low energy becomes $\left\langle y y^{\prime}\right\rangle$ focusing.

The advantage of weak antisolenoids is that they compensate the effects of the detector solenoid on the IP beam size almost completely, and the major coupling terms are

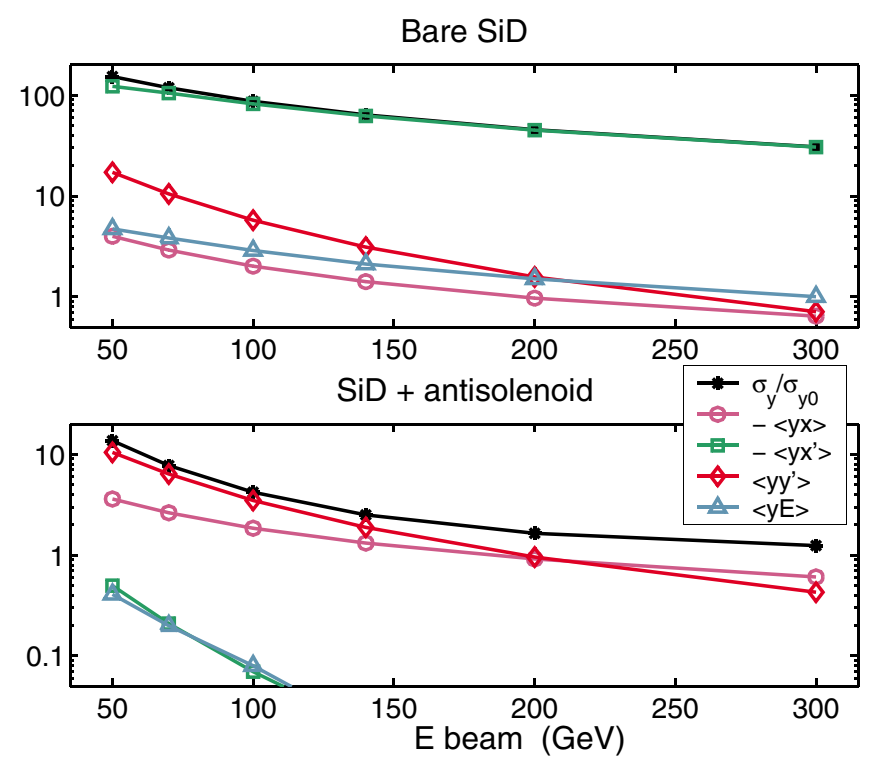

FIG. 18. (Color) Beam size and beam distortions in $\mathrm{SiD}$, as a function of beam energy without and with antisolenoid. Linear correction knobs have not been applied. practically canceled for any beam energy. Figure 18 (bottom plot) shows the IP beam size and distortions for the $\mathrm{SiD}$ with the antisolenoid. The antisolenoid compensates more than $99 \%$ of the solenoid effect at $300 \mathrm{GeV}$ and more than $90 \%$ at $50 \mathrm{GeV}$.

For $\mathrm{SiD}$ with the antisolenoid, the remaining linear distortions can be corrected by linear knobs. At the lowest beam energy, the quadrupoles QD0 and QF1 were adjusted to compensate the solenoid focusing term $\left\langle y y^{\prime}\right\rangle$, skew quadrupoles in the skew correction section were used to correct the $\langle y x\rangle$ term, and sextupole displacement knobs were used for fine adjustment of $\langle y E\rangle,\langle x E\rangle$, and $\left\langle y x^{\prime}\right\rangle$. The beam size at the IP is shown in Fig. 19 for $\mathrm{SiD}$ plus antisolenoid and corrected using only these knobs. The beam size correction is better than $10 \%$ at $100 \mathrm{GeV} /$ beam, and within $35 \%$ at $50 \mathrm{GeV}$. The remaining increase of the beam size is due to higher order distortions, in particular, the second order coupling term $\left\langle y x^{\prime} x^{\prime}\right\rangle$ shown in Fig. 20, which arise because the linear knobs such as $\langle y x\rangle$ needed at low energy are too strong. Further correction of the beam size at low energy requires tuning with higher order knobs. For example, knobs acting on $\left\langle y x^{\prime} x^{\prime}\right\rangle$ and $\langle y E E\rangle$ require rotation of the BDS sextupoles. Other second order knobs also adjust the sextupole strength. A discussion of this procedure is beyond the scope of this paper and can be found in [21].

The performance of solenoid compensation using weak antisolenoids was compared with compensation using skew quadrupoles in the FD, for the $\mathrm{SiD}$ detector. The best results with skew quadrupoles were obtained not with the skew quadrupole SQ3 located next to the FD, but with a rotation of QD0, which gives a more local correction of the $\left\langle y x^{\prime}\right\rangle$ coupling.

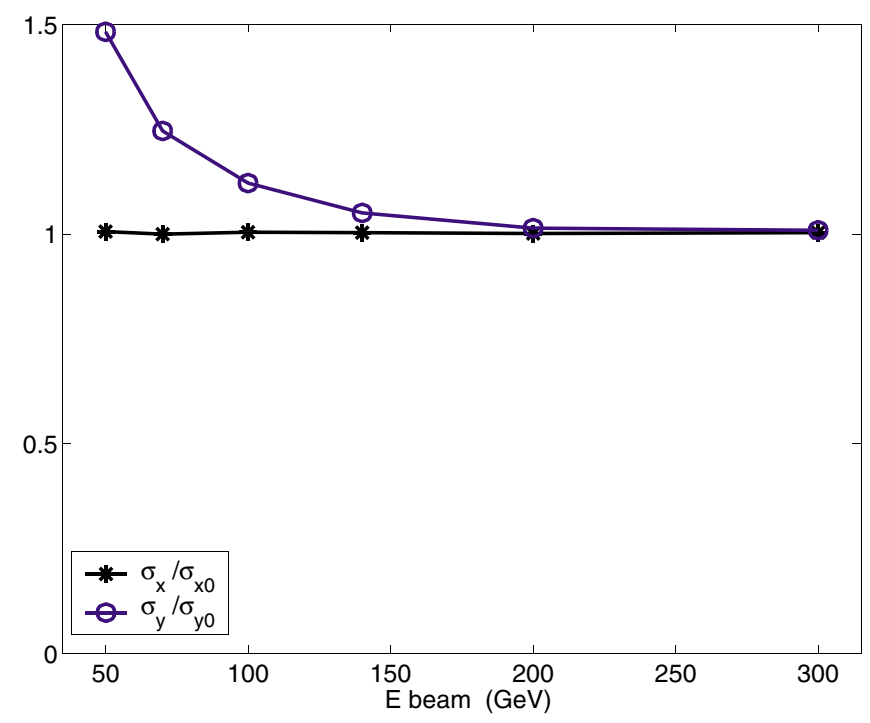

FIG. 19. (Color) Beam size in $\mathrm{SiD}$ with the antisolenoid as a function of beam energy. The beam size is corrected only with linear knobs. 


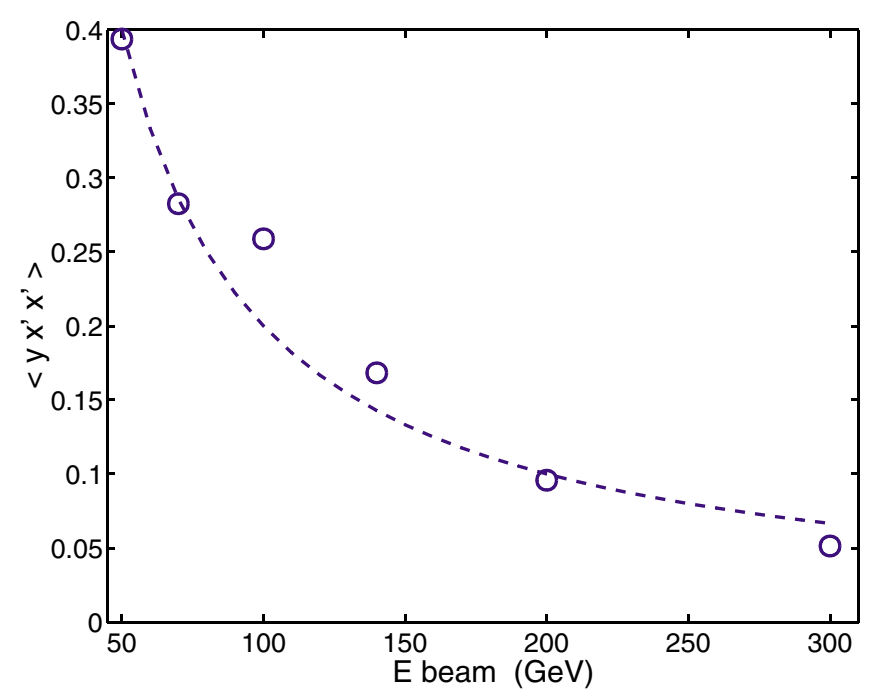

FIG. 20. (Color) One of the remaining second order terms at the IP, $\left\langle y x^{\prime} x^{\prime}\right\rangle$, for the SiD model with an antisolenoid corrected only with linear knobs.

Using only the quadrupole rotation, the IP vertical orbit is not automatically compensated. To cancel the IP orbit requires offsets of QD0 and QF1 and an additional dipole corrector (BXCOR in Fig. 2) located between 6.6 and $7.6 \mathrm{~m}$ in the FD. These three dipole correctors cancel both the vertical dispersion and vertical offset at the IP, without introducing a second order vertical dispersion.

The rest of the distortions were compensated as for the antisolenoid case, adjusting the QD0 and QF1 strength to correct $\left\langle y y^{\prime}\right\rangle$, and the BDS skew correction to cancel $\langle y x\rangle$. Using the QD0 rotation and these linear knobs, the vertical beam size was corrected to $\sigma_{y} / \sigma_{y 0}=1.71$ at $50 \mathrm{GeV}$, in comparison with the $\sigma_{y} / \sigma_{y 0}=1.48$ that was achieved with the antisolenoid.

This suggests that, though the weak antisolenoid correction gives better performance, the skew quadrupole method may also be feasible, although it would require a more sophisticated linear and higher order tuning. However, one of the disadvantages of the rotated quadrupole method is that changing the beam energy would require either mechanical rotation of QD0 or adjustment of its skew winding, if it is superconducting, as well as a readjustment of all the knobs.

In the case of the weak antisolenoids, the fact that the compensation is largely independent of beam energy is an advantage that would significantly ease operation of the machine.

Finally, the effect of the crab cavity on the solenoid compensation and beam size at the IP has been investigated and found negligible. This cavity is located about $12 \mathrm{~m}$ from the IP, and produces a horizontal beam kick correlated with the longitudinal position within the bunch. As a result, the beam at the IP becomes tilted in the $x-z$ plane by the angle $\theta_{c}$, providing the nominal head-on collision with the opposite beam. Because of the detector solenoid coupling, the crab-cavity kick would also cause a $y$ - $z$ tilt at the IP which is easily corrected by rotation of the crab cavity about the longitudinal axis. Once this (energy dependent) correction is made, the effect of the crab cavity on the beam size vanishes.

\section{CONCLUSION}

The solenoid field of a linear collider experimental detector will distort the beam size at the interaction point unless compensated. This paper discusses optics perturbations at the IP caused by the realistic detector solenoid field, and the performance of various methods of compensation. Most of the effect on beam size is caused by the part of the solenoid field which overlaps and extends beyond the final focusing quadrupoles. It is shown that the most efficient and most local compensation is achieved with weak antisolenoids placed near the overlapped quadrupoles. A compensation scheme using only skew quadrupoles is found less efficient but also feasible. The important advantage of the antisolenoid method is that the compensation is effective over a large beam energy range.

\section{ACKNOWLEDGMENTS}

The authors thank John Hodgson for providing ANSYS models of the NLC detectors, Peter Tenenbaum for developing the DIMAD models of BDS with overlapping solenoid field, and Brett Parker, Nan Phinney, and Tor Raubenheimer for very useful discussions. The work was supported by the U.S. Department of Energy, Contract No. DE-AC02-76SF00515.

[1] J. J. Murray, SLAC Note No. PEP-219, 1976.

[2] KEK-B B-Factory Design Report No. 95-7, 1995.

[3] M. Bassetti, M. E. Biagini, and C. Biscari, in Proceedings of the Advanced ICFA Beam Dynamics Workshop, Frascati, Italy, 1997, edited by L. Palumbo and G. Vignola (Instituto Nazionale de Fisica Nucleare, Frascati, 1998), p. 209.

[4] LEP Design Report No. CERN-LEP-84-01, 1984, Vol. 2.

[5] Y. Nosochkov, Y. Cai, J. Irwin, M. Sullivan, and E. Forest, in Proceedings of the 1995 Particle Accelerator Conference, Dallas, 1995 (IEEE, Piscataway, NJ, 1995), p. 585.

[6] A. Seryi, Y. Nosochkov, and M. Woodley, in Proceedings of the 2004 European Particle Accelerator Conference, Lucerne, 2004 (EPS-AG and CERN, Geneva, 2004).

[7] P. Raimondi and A. Seryi, Report No. SLAC-PUB-8460, 2000; Phys. Rev. Lett. 86, 3779 (2001).

[8] R. Talman, in Frontiers of Particle Beams: Observation, Diagnosis and Correction, edited by M. Month and S. Turner, Lecture Notes in Physics Vol. 343 (SpringerVerlag, Berlin, 1989), p. 42. 
[9] J. J. Murray, SLAC Note No. PEP-256, 1977.

[10] S. Peggs, Report No. CERN/SPS/82-2 (DI/MST), 1982.

[11] R. H. Helm, Report No. SLAC-0004, 1962.

[12] P. Tenenbaum, J. Irwin, and T. O. Raubenheimer, Phys. Rev. ST Accel. Beams 6, 061001 (2003).

[13] A. Wolski, TESLA Report No. 2000-13, 2000.

[14] J. J. Murray, Report No. SLAC-CN-237, 1983.

[15] O. Napoly, E. Klein, and J.M. Rifflet, Report No. DAPNIA-94-10, 1994.

[16] P. Tenenbaum, SLAC Report No. LCC-Note-020, 1999.

[17] R. Servranckx, K. Brown, L. Schachinger, D. Douglas, and P. Tenenbaum, Report No. SLAC-285, 1990; http:// www. slac . stanford. edu/ accel/nlc / local/ AccelPhysics/ codes/dimad/dimad.pdf

[18] B. Parker and A. Seryi, Report No. SLAC-TN-04-044, 2004.

[19] Y. Nosochkov and A. Seryi, Report No. SLAC-PUB10592, 2004.

[20] KEK Report No. 2003-7, edited by N. Toge, 2003.

[21] Y. Nosochkov, P. Raimondi, T. O. Raubenheimer, A. Seryi, and M. Woodley, in Proceedings of the 8th European Particle Accelerator Conference, Paris, 2002 (EPS-IGA and CERN, Geneva, 2002). 